\title{
An Identified Interneuron Contributes to Aspects of Six Different Behaviors in Aplysia
}

\author{
Yuanpei Xin, ${ }^{1}$ Klaudiusz R. Weiss, ${ }^{2}$ and Irving Kupfermann ${ }^{1}$ \\ ${ }^{1}$ Center for Neurobiology and Behavior, College of Physicians and Surgeons, Columbia University, New York, New York, \\ 10032, and 'Department of Physiology and Biophysics, Mount Sinai Medical Center, New York, New York 10029
}

Previous results have indicated that the bilateral cerebral interneuron CC5 mediates the pedal artery shortening that is a component of defensive withdrawal responses involving the head. Current studies suggest that CC5 contributes to aspects of at least six different behaviors: locomotion, head turning, defensive head withdrawal, local tentacular withdrawal, rhythmic feeding, and head lifting. In addition to receiving input from mechanoreceptors in the head, CC5 receives synaptic input during fictive locomotor and feeding programs. Firing of CC5 produces widespread monosynaptic or polysynaptic actions in all ganglia in the animal. CC5 excites presumptive motor neurons for the neck, and its activity can contract neck muscles. The pedal artery shortener motor neuron (PAS), a key excitatory follower cell of CC5, fires during ipsilateral head turning, head withdrawal, tentacle withdrawal, feeding, and locomotion. For all behaviors, except locomotion and biting, responses of PAS were eliminated by cutting the ipsilateral-pleural connective, which interrupts the only direct connection of CC5 to the ipsilateral PAS. The data suggest that CC5 is a multifunctional interneuron that plays different roles during different behaviors. The neuron appears to be involved in producing coordinated movements of the head, involving both somatic and visceral muscles. For some behaviors, or for certain aspects of behaviors, CC5 appears to act as an individual command-like neuron; for other behaviors, CC5 appears to act more as an element of a distributed circuit and is neither necessary nor sufficient for any aspects of the behavior.

Key words: command; withdrawal reflex; Aplysia; mechanosensory; feeding; head turning; locomotion
An important conceptual issue in understanding the organization of the nervous system relates to how information is represented. To what extent is information encoded by the activity of individual neurons that perform dedicated functions using local-coding or are neurons always a part of distributed networks using vector coding in which their functional roles are determined by the activity of other neurons in the network (Barlow, 1972; Churchland and Sejnowski, 1992; Morton and Chiel, 1994)? A related question is whether neurons are multifunctional, and growing evidence suggests that indeed multifunctional neurons may be commonly used by the nervous system (Hooper and Moulins, 1989; Lockery and Kristan, 1990; Fetz, 1992; Meyrand et al., 1994; Wu et al., 1994). What is unclear is how the activity of multifunctional neurons is translated into behavior and whether multifunctional neurons can serve different roles for different behaviors. In a companion paper, we describe an identified interneuron, CC5, that appears to be necessary and sufficient for the arterial artery shortening component of a local withdrawal response (Xin et al., 1996) (see also Skelton and Koester, 1992). In the present paper, we describe data that indicate CC5 is engaged in multiple behaviors, all of which involve movements of the neck of the animal. Although one or both CC5 neurons contribute to components of these behaviors, the precise role of the cells is different for each of the behaviors.

\footnotetext{
Received Feb. 16, 1996; revised June 12, 1996; accepted June 14, 1996.

This work was supported in part by National Institutes of Health Grants MH36730, GM32099, and MH35564. We thank S. C. Rosen for comments on this manuscript.

Correspondence should be addressed to Irving Kupfermann, Center for Neurobiology and Behavior, College of Physicians and Surgeons, Columbia University, 722 West 168th Street, Research Annex, New York, NY 10032.

Copyright (C) 1996 Society for Neuroscience $0270-6474 / 96 / 165266-14 \$ 05.00 / 0$
}

\section{MATERIALS AND METHODS}

The animals and methods in these experiments were similar to those described in detail in a previous paper (Xin et al., 1996). Unless otherwise mentioned, in all experiments studying the connections between neurons, we used the isolated head ganglia preparation (buccal, cerebral, and pedal-pleural) interconnected by their connectives.

\section{RESULTS}

\section{CC5 receives inputs from sources other than the lips} and tentacles

We reported previously that $\mathrm{CC} 5$ is excited by tactile stimuli applied to the lips and tentacles. To determine if CC5 receives inputs from other sources, we used electric stimulation of nerves. We found that stimulation of buccal nerves excited CC5 (Fig. $1 A 1)$. The excitation was not eliminated when the ganglia were placed in a high-divalent solution. Recordings from the pedal artery nerve (PAn) showed increased firing of a unit whenever the firing rate of CC5 increased. Previous data (Xin et al., 1996) strongly suggest that the unit in the nerve reflects the firing of the pedal artery shortener motor neuron (PAS), a cell that is strongly excited by $\mathrm{CC} 5$. In preparations that included the buccal mass, mechanical stimulation of the buccal mass evoked several cycles of buccal mass movements, and CC5 was excited during each cycle (Fig. 1 $A 2$ ) at the phase in which the buccal mass visibly moved backward and the radula retracted.

To study the possible activity of CC5 during locomotion, we stimulated a pedal nerve (P9) that is known to initiate a fictive locomotor program (Hening et al., 1979; Fredman and JahanParwar, 1983) and that exhibits activity in phase with normal locomotion (Fig. 1B1). Stimulation of P9 caused an initial inhibition of $\mathrm{CC} 5$, followed by several cycles of bursts of firing of CC5 in phase with bursts in the $\mathrm{P} 10$ nerve, suggesting that $\mathrm{CC} 5$ receives 
A1
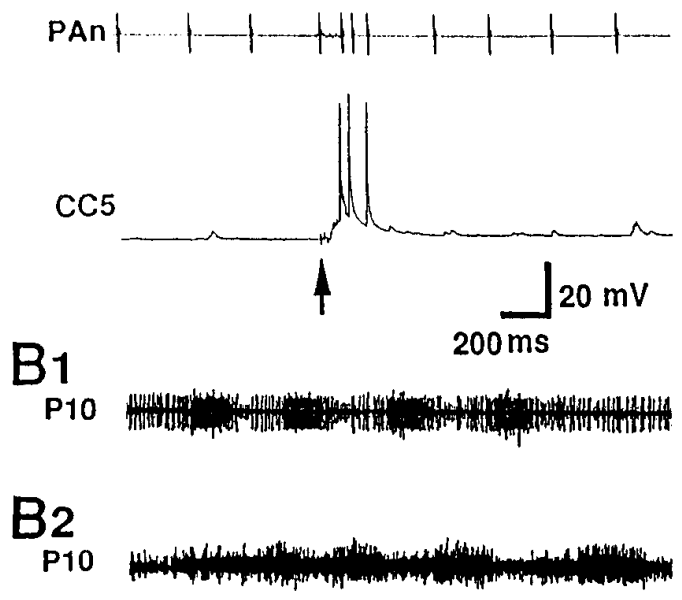

PAn
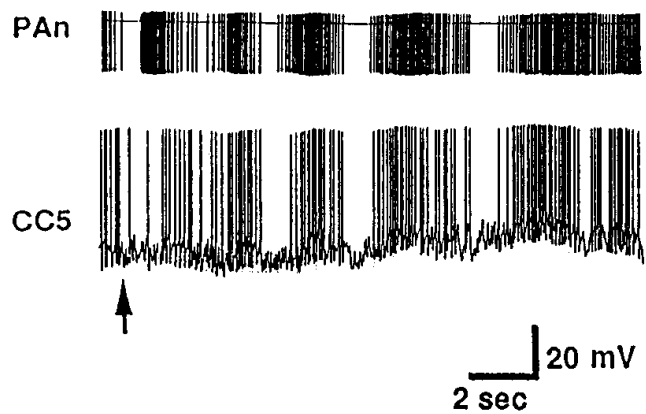

A2
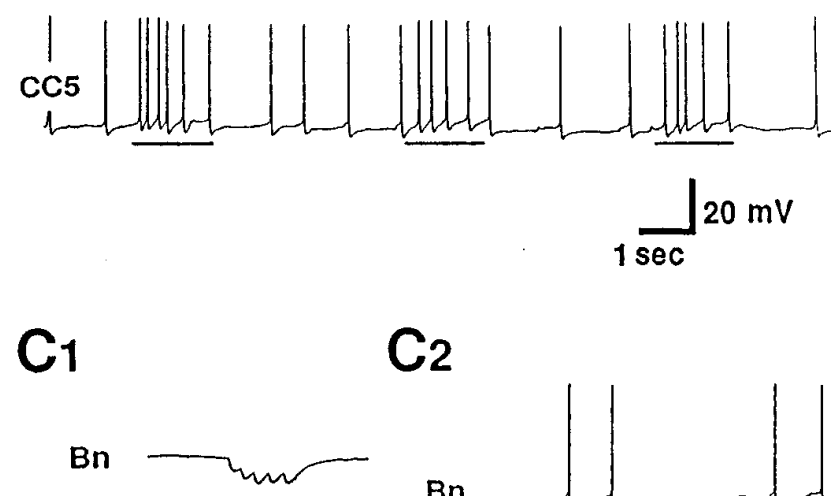

$\mathrm{C}_{2}$

$\mathrm{Bn}$
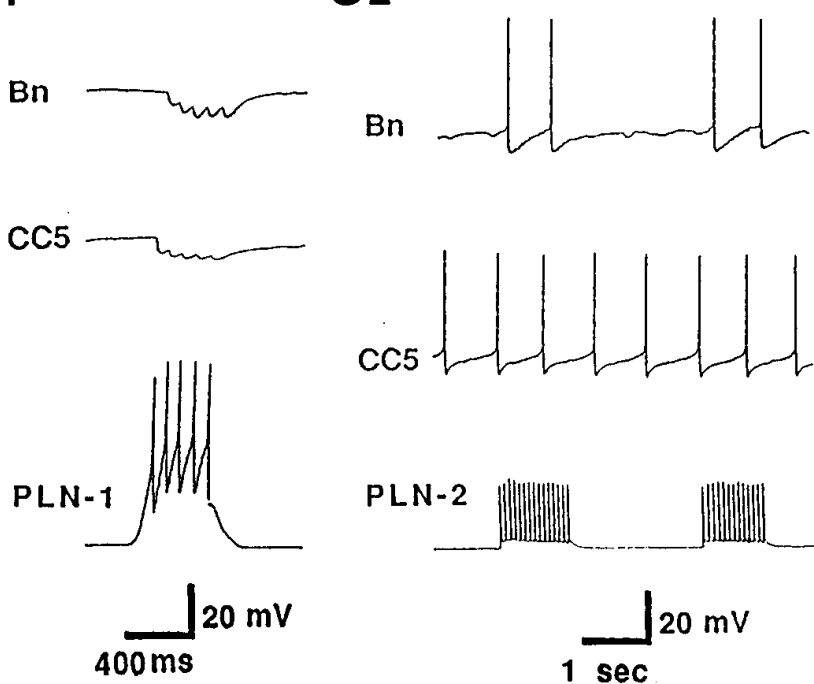
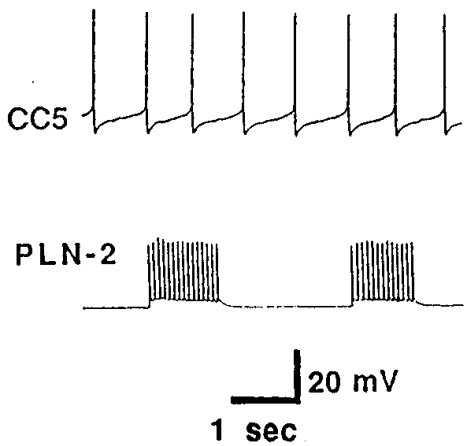

Figure 1. Inputs to CC5. A, Inputs from the feeding system. $A 1$, In a preparation in which the buccal ganglion remained attached to the cerebral ganglion, electrical stimulation (shocks, at arrow) of buccal nerve 2 evoked excitatory synaptic input to CC5 and increased the activity of the excitatory follower cells of CC5, the PAS. Activity of PAS was recorded extracellularly from the pedal artery nerve $(P A n)(n=6) . A 2$, In a preparation in which the buccal ganglion and the buccal mass remained attached to the cerebral ganglion, spontaneous rhythmic movements of the buccal mass were associated with increased excitatory input into $\mathrm{CC} 5$, in phase with retraction phase of radula movement (lines below trace) $(n=5)$. $B$, CC5 receives synaptic rhythmic input during a fictive locomotor program elicited by electrical stimulation of pedal nerve 9 (P9). B1, In intact animals, an extracellular recording from P10 exhibits bursts of unit activity that are in phase with the neck shortening phase of a locomotor step (monitored visually) that occurred spontaneously or was elicited by a tail pinch $(n=6)$. B2, During a fictive locomotor program elicited by stimulation of the P9 nerve in the isolated nervous system, rhythmic bursts of activity occur in P10, and the bursts are in phase with firing of CC5 and of the PAS neurons as monitored in the PAn $(n=7)$. $C$, CC5 receives inhibitory synaptic inputs from a class of interneurons located in the pleural ganglion. $C 1$, Inhibitory input to CC5 from a pleural interneuron (PLN-1) that inhibits cerebral Bn cells. C2, Lack of synaptic input to CC5 from a pleural interneuron (PLN-2) that excites Bn cells $(n=6)$.

rhythmic excitation during a locomotor program that is expressed in the nerve.

CC5 was also found to receive inhibitory synaptic inputs from a subpopulation of pleural interneurons (Fredman and JahanParwar, 1979), which also inhibit the cerebral B neurons (Fig. 1C1). Based on their constant latency, the connections to CC5 and to the B neurons appear to be monosynaptic. Another subpopulation, which excites the cerebral B neurons, was found to have no inputs to CC5 (Fig. 1C2). There is evidence that pleural interneurons that inhibit cerebral $\mathrm{B}$ neurons contribute to certain phases of locomotor movements (Jahan-Parwar and Fredman, 1979). Thus, the relationship between $\mathrm{CC} 5$ and the pleural inhibitory neurons supports the idea that CC5 may be involved in locomotion.

\section{CC5 affects the activity of neurons that send axons into the pleural and pedal nerves, and the cerebral- buccal and pleural-abdominal connectives}

Because CC5 previously was found to receive differential sensory input from the ipsilateral and contralateral regions of the head
(Xin et al., 1996), we examined the possibility that CC5 may be involved in asymmetric head movements, such as those that occur during head waving and directed head turning. Accordingly, we examined whether firing of CC5 could affect the activity of units recorded from pedal and pleural ganglion nerves that control various regions of the neck and foot. It was previously found that extracellular recordings from pedal and pleural nerves and from neck muscles in free-moving animals show increases of activity when a head turn occurs spontaneously or in response to contact with a piece of food (Bablanian et al., 1987; Cook and Carew, 1989; Teyke et al., 1990a; Kuenzi and Carew, 1994a). The nerves and muscle exhibited an increase of activity when the animal turned in the ipsilateral direction and often showed a decrease of activity when the animal turned in the contralateral direction. In the current experiments, we recorded simultaneously from the left and right pleural nerve 1 (Fig. $2 A$ ) and pedal nerves 4,5 , and 7 (Fig. $2 B, C, D$ ) in isolated head ganglia preparations. When CC5 was fired by means of intracellular depolarizing current, there was increased unit activity in the ipsilateral nerves, but little or no 
A

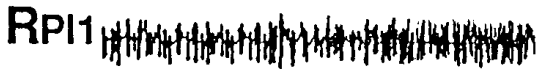

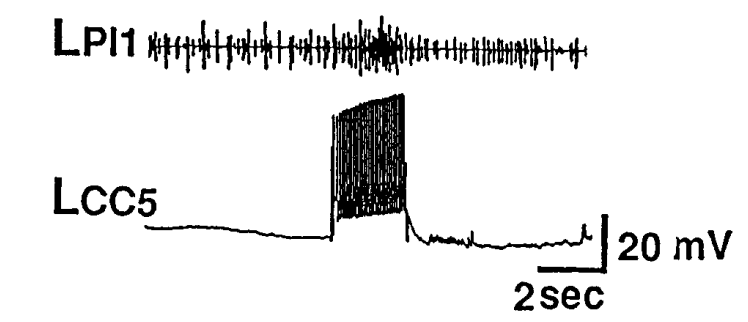

B

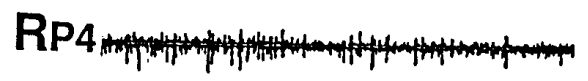

LP4

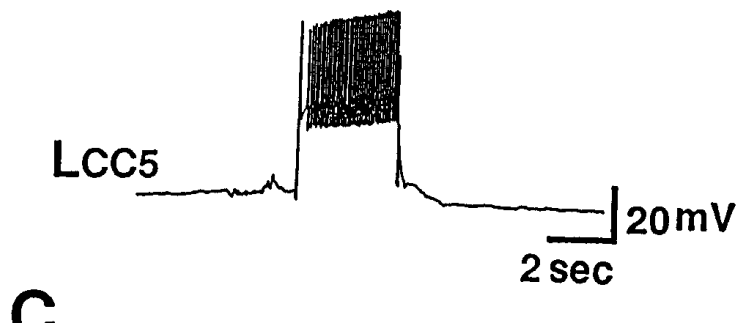

C

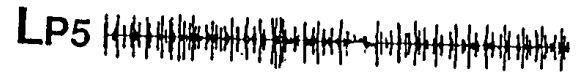

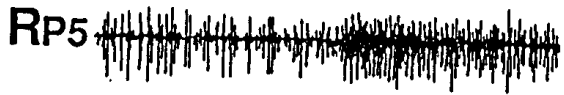

D

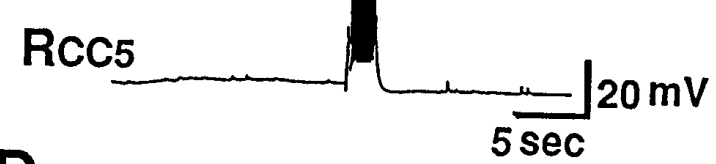

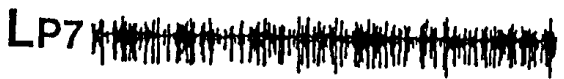
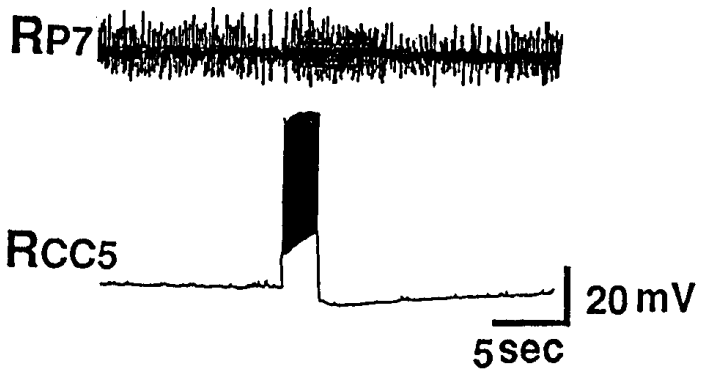

Figure 2. Effects of firing of CC5 on unit activity in nerves that innervate the neck and foot musculature. A single CC5 was impaled, and simultaneous recordings from the ipsilateral and contralateral nerves were obtained. $A$, Firing CC5 excited units in the ipsilateral PL1 nerve and had no effect or slightly inhibited units in the contralateral PL1 nerve $(n=10)$. $B-D$, Firing $C C 5$ excited units in the ipsilateral P4, P5, and P7 nerves and had no effect or an inhibitory effect in the contralateral nerves $(n=10)$.

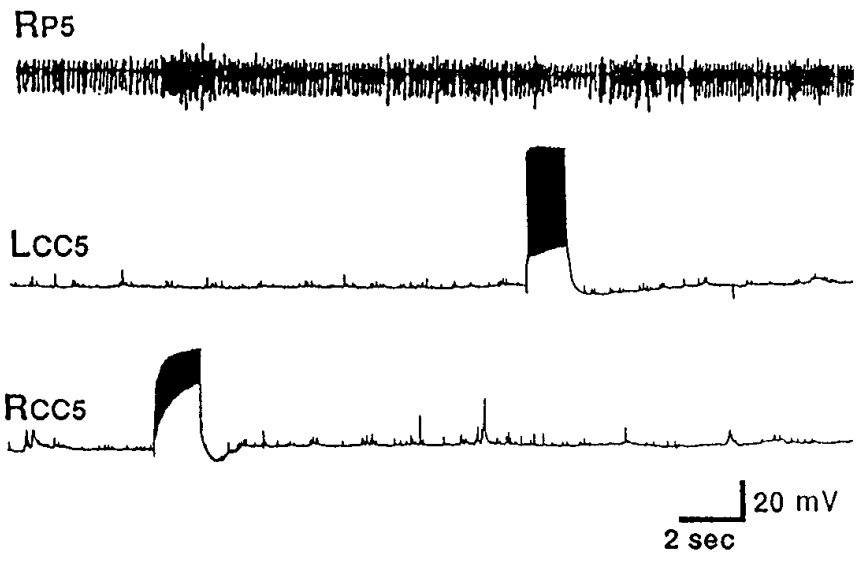

Figure 3. Simultaneous recordings from the left and right CC5 showed that firing of the ipsilateral CC5 excited spike activity in the P5 nerve, whereas firing of the contralateral CC5 inhibited spike activity in the P5 nerve $(n=5)$.

change or decreased activity in the contralateral nerves. In experiments in which we recorded from one P5 nerve and impaled both the ipsilateral and contralateral $\mathrm{CC} 5$, we found that firing of only the ipsilateral CC5 excited spike activity in the P5 nerve (Fig. 3), whereas firing of the contralateral CC5 clearly inhibited spike activity in the P5 nerve (see also Fig. 7A,B). Note that the two CC5 cells have little or no synaptic effects on the contralateral cell and that they do not have any obvious common spontaneous synaptic inputs. These results suggest that the two CC5 cells can act independently and can have differential effects on the outputs of the ipsilateral and contralateral neurons that send axons into the pedal and pleural nerves.

We found that $\mathrm{CC} 5$ altered the activities of neurons that project into connectives that interconnect the central ganglia, including the cerebral-buccal connective (CBC) and the pleural-abdominal connective. These data suggest that CC5 may affect not only pedal and pleural ganglia neurons, but also buccal and abdominal ganglion neurons. Firing of the ipsilateral and contralateral CC5 cells in the same preparation (Fig. 4) revealed that both cells had effects on units that project into the pleural-abdominal connectives. When both cells were fired at the same rate $(15 \mathrm{~Hz}, 2 \mathrm{sec}$; Fig. 4), a stronger action was produced by the ipsilateral cell. Firing of CC5 also increased the activity of at least one unit that projects from the cerebral ganglion into the $\mathrm{CBC}$ (Figs. 5A, 13). The unit activity evoked by $\mathrm{CC} 5$ was consistent within a preparation, but was somewhat variable from preparation to preparation. The effect of $\mathrm{CC} 5$ on the $\mathrm{CBC}$ unit was blocked when the axon of CC5 that travels out of the cerebral ganglion to the pedal-pleural ganglion via the cerebral-pleural (C-PL) connective was interrupted by cutting the C-PL connective [for a diagram of the course of the CC5 axon into the connectives, see Xin et al. (1996), their Fig. 2]. Because the effects of $\mathrm{CC} 5$ on the $\mathrm{CBC}$ were also blocked by severing the cerebral-pedal connective, it is likely that $\mathrm{CC} 5$ acts by activating an interneuron that is located in the pedal or pleural ganglion and that projects into the cerebral-pedal connective. The putative interneuron may synaptically activate a cerebral-buccal interneuron because the capacity of CC5 to evoke activity in the $\mathrm{CBC}$ was also blocked (Fig. 5A,B) when the cerebral ganglion (but not the pedal-pleural ganglion) was placed in a solution $(4 \times$ $\left.\mathrm{Mg}^{2+}, 0.1 \times \mathrm{Ca}^{2+}\right)$ that eliminates chemical synaptic potentials. When the blocking solution was removed from the cerebral ganglion, responses at least partially recovered (Fig. 5C). 


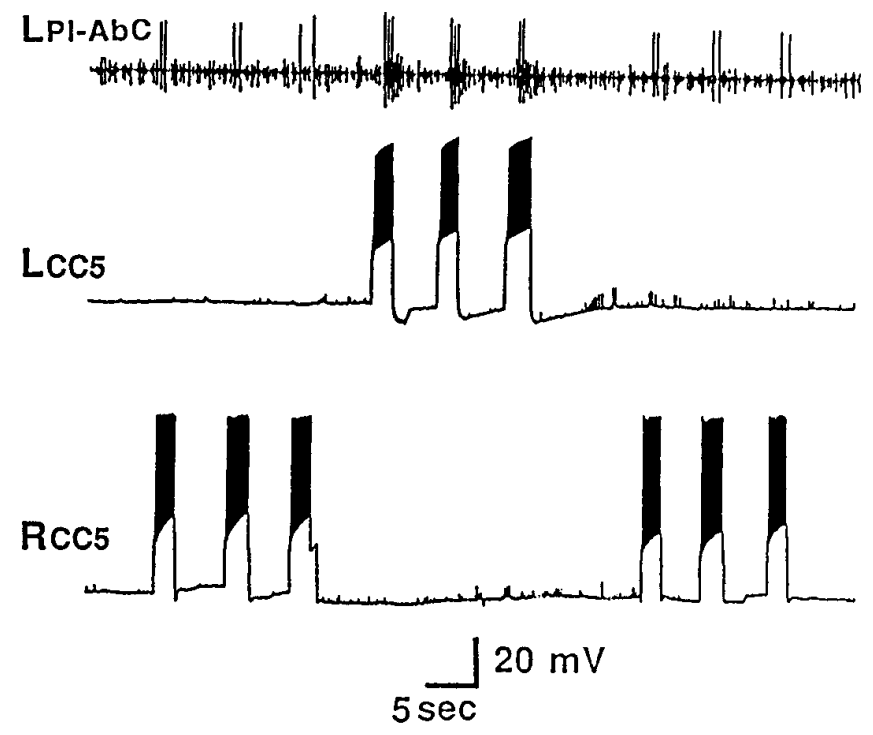

Figure 4. Effect of firing CC5 on unit activity recorded from the pleural ganglion end of the pleural-abdominal connectives. The ipsilateral and contralateral CC5 were recorded simultaneously in the same preparation. A large unit was found to be excited by firing both the ipsilateral and contralateral CC5. The ipsilateral action was stronger, and there was some indication of excitation of more than one unit $(n=5)$.

\section{Effects of CC5 on neck muscles}

The neck muscles of Aplysia contain longitudinal, transverse, oblique, and helical fibers (Bablanian et al., 1987; Cook and Carew, 1989; Nagahama et al., 1993). During head and neck turning movements, electromyographic activity in these muscles can be recorded (Cook and Carew, 1989; Teyke et al., 1990a; Kuenzi and Carew, 1994a), indicating that head movements may involve a complex interaction of the contraction of different muscle groups. To explore the possible involvement of CC5 in head turning, we examined its effect on neck muscles. In semi-intact preparations, we found that firing of CC5 evoked a robust ipsilateral contraction (Fig. 6A1) and a weaker contralateral contraction (Fig. 6A2) of neck muscles. Contractions occurred in both the longitudinal and transverse directions, but the transverse contractions were considerably weaker (Fig. 6A1,B1,A2,B2). Furthermore, transverse contractions were considerably more spatially limited, occurring primarily at the region in which the pedal artery enters the body wall. Because CC5 does not send an axon into a peripheral nerve, its effect on neck muscle contractions is presumably mediated by other neurons in the pleural-pedal ganglia that are excited by CC5 and send axons into pedal and pleural nerves. Consistent with this interpretation was the observation that the effects of CC5 on contraction of neck muscles was reversibly eliminated when the head ganglia alone were bathed in a highdivalent cation solution $\left(5 \times \mathrm{Ca}^{2+}, 2 \times \mathrm{Mg}^{2+}\right)$, which raises neuronal thresholds. Experiments described in a later section directly demonstrated that CC5 excites motor neurons innervating the neck.

\section{Four populations of pedal neurons respond to firing of the ipsilateral and contralateral CC5}

Morphological studies of CC5 indicate that it projects not only to the ipsilateral pedal-pleural ganglia, but also sends an axon into the pedal commissure to the contralateral ganglia (Xin et al., 1996). Extracellular nerve recordings also revealed ipsilateral and contralateral synaptic effects. To begin to explore the functional
A
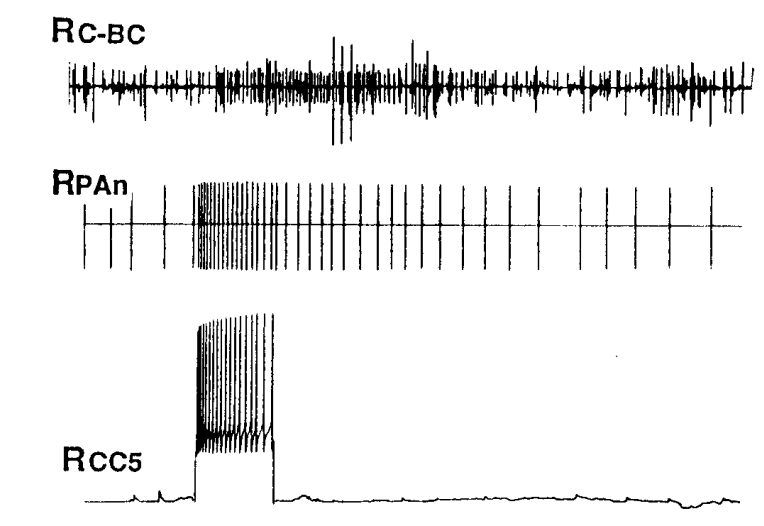

B
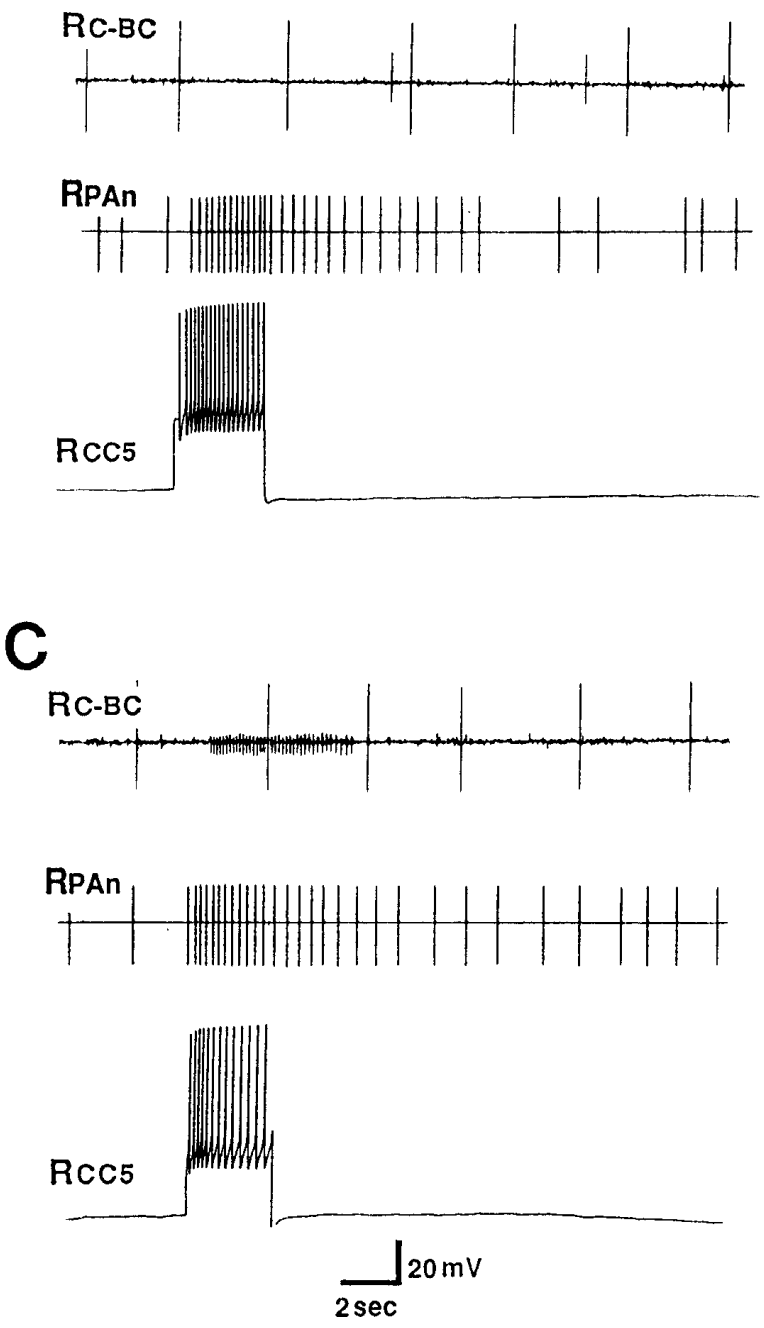

Figure 5. Effect of firing CC5 on unit activity recorded from the cerebral ganglion end of the ipsilateral CBC. CC5 was identified as usual by its effect on the PAS as recorded in the ipsilateral PAn. $A$, In normal ASW, firing of CC5 evoked a burst of activity in the CBC. B, CC5 no longer evoked activity when the cerebral ganglion was bathed in a solution that blocks chemical synapses $\left(4 \times \mathrm{Mg}^{2+}, 0.1 \times \mathrm{Ca}^{2+}\right)$. The effect on PAS, which is located in the pedal ganglion, shows little or no change. $C$, The capacity of $\mathrm{CC} 5$ to evoke unit activity in the $\mathrm{CBC}$ recovers when the cerebral ganglion is washed in ASW $(n=6)$. 
A

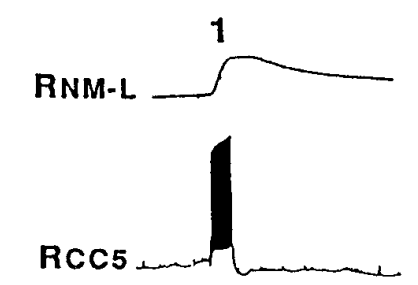

B

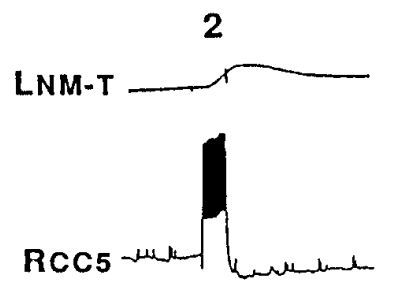

1

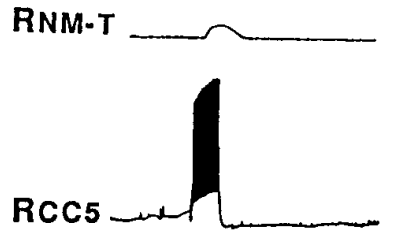

2

LNM-T

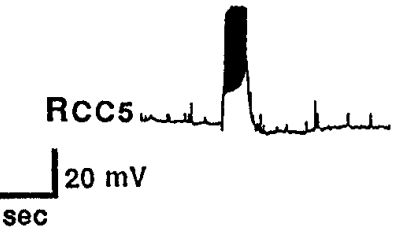

Figure 6. Isotonic tension records showing that firing of CC5 could evoke contractions of muscles in different regions of the neck. Bilateral pleural nerve 1 , pedal nerves 4,5 , and 7 , and the neck muscles they innervate were intact. The right CC5 was impaled, and a force transducer was placed to selectively record movements in the transverse or longitudinal direction (indicated by $L$ or $T$ suffix) of the ipsilateral (right) or contralateral (left) muscle (indicated by $R$ or $L$ prefix). For each trial, CC5 was fired at $\sim 15$ $\mathrm{Hz}$ for $2 \mathrm{sec}$. A1, Longitudinal contractions in the ipsilateral neck muscles $(R N M-L) . A 2$, Transverse contractions in the contralateral neck muscles $(L N M-T) B 1, B 2$, Transverse contractions of the ipsilateral $(R N M-T)$ or the contralateral $(L N M-T)$ neck muscles near the region where the pedal artery enters the muscle $(n=5)$.

significance of these bilateral connections, we canvassed the pedal ganglion to locate and characterize neurons receiving inputs from CC5. Four regions, defined previously (Hening et al., 1979), were found to contain cells that responded to the ipsilateral, contralateral, or both CC5 neurons. Two groups of neurons (PIIa and PIIb) were located in region II. Cells in the PIIa class were excited by the ipsilateral $\mathrm{CC} 5$ and were inhibited by the contralateral CC5 (Fig. 7A). Cells in the PIIIa class had responses opposite to those of the PIIa class of cells (Fig. $7 B$ ). PIIb neurons also were excited by the ipsilateral CC5 (Fig. 7C), but the contralateral CC5 had little or no effect on these neurons. It is not clear whether the PIIa and PIIb neurons fall along a continuum or are fully distinct classes. Finally, cells located in regions Ia and Ib of the pedal ganglion (PI cells) were found to receive inhibitory synaptic inputs from the ipsilateral CC5 (Fig. 7D). We intracellularly labeled selected neurons from all groups and found that they all have axons that project into pedal nerves.

\section{CC5 evokes a monosynaptic EPSP in some putative neck motor neurons in the pedal ganglion}

Many of the pedal neurons affected by firing of CC5 appeared to receive polysynaptic inputs as judged by their inconstant latency size. We also obtained evidence, however, that some neurons receive monosynaptic input. The results of recordings from two

A
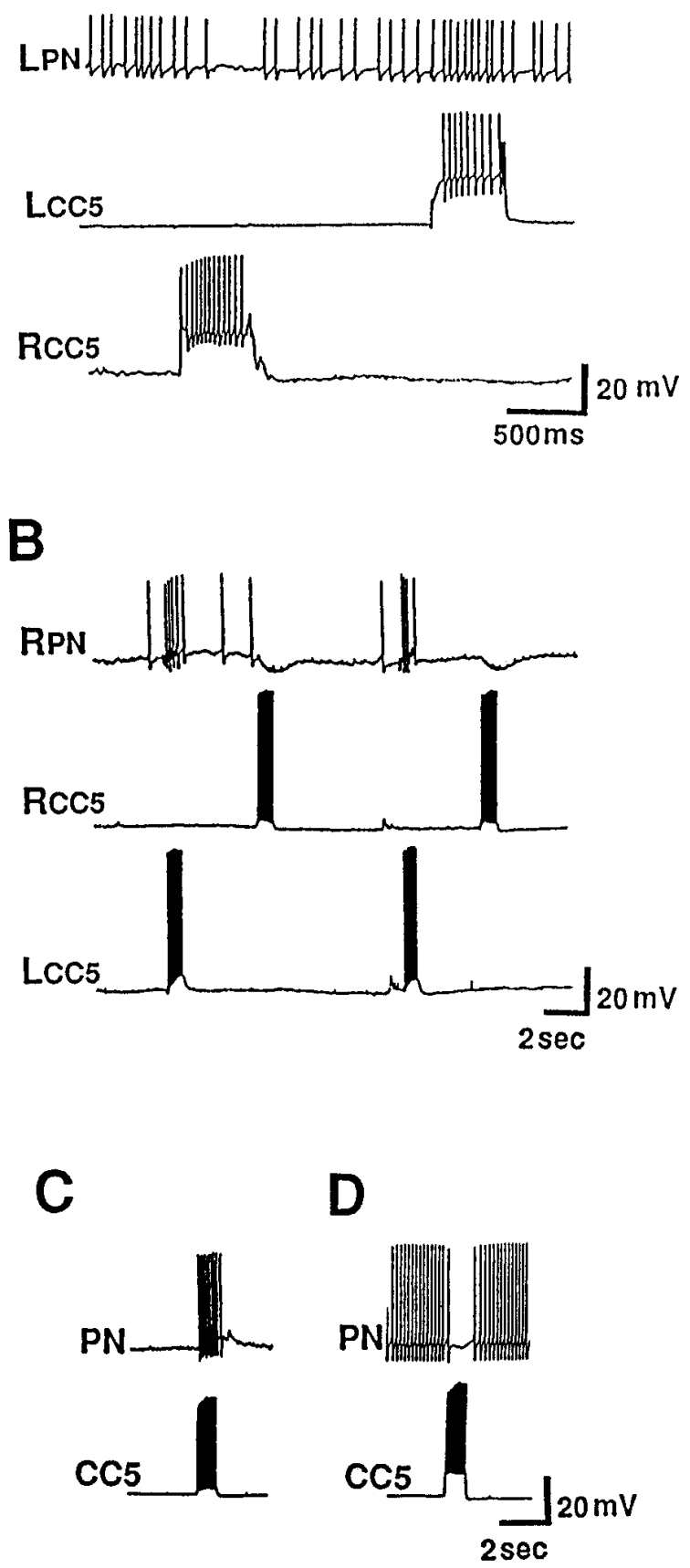

Figure 7. Effects of firing CC5 on neurons in the pedal ganglion. Four types of neurons, based on their responses to the ipsilateral and contralateral CC5 input, could be distinguished (see text). $A$, Response of group IIa neurons. $B$, Response of group IIIa neurons. $C$, Response of group IIb neurons. $D$, Response of group Ia and Ib neurons $(n=14)$.

such neurons recorded in the same preparation from region II are illustrated in Figure 8. Dye fills showed that each neuron sent a single process to the P5 nerve or a sub-branch of P5 (Fig. 8A1, $A 2)$. Each neuron received a short-latency EPSP from the ipsilateral CC5 neuron (Fig. $8 B 1 a, B 1 b$ ), and the EPSPs persisted in a high-divalent cation solution (Fig. $8 B 2 a, B 2 b$ ). Firing of the neurons evoked small but distinct longitudinal contractions of the neck (Fig. 8C1,C2). 
A1

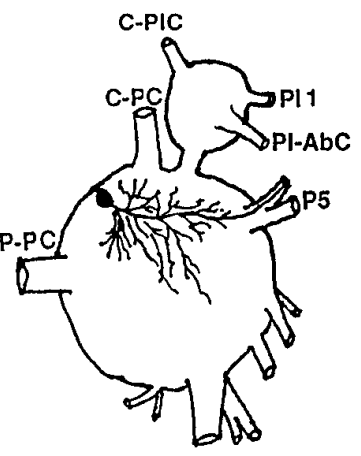

B1a

normal ASW

PN1

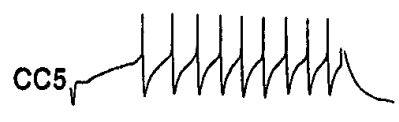

$B_{1 b}$

$\mathrm{B} 2 \mathrm{~b}$
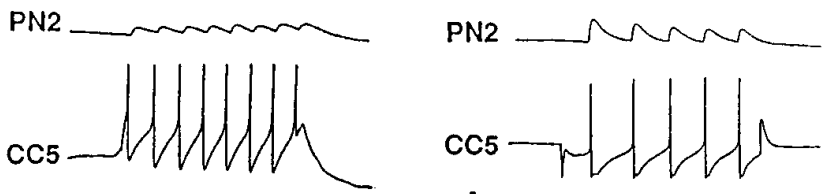

$20 \mathrm{mV}$

$200 \mathrm{~ms}$

$\mathrm{C}_{1}$

$\mathrm{C} 2$
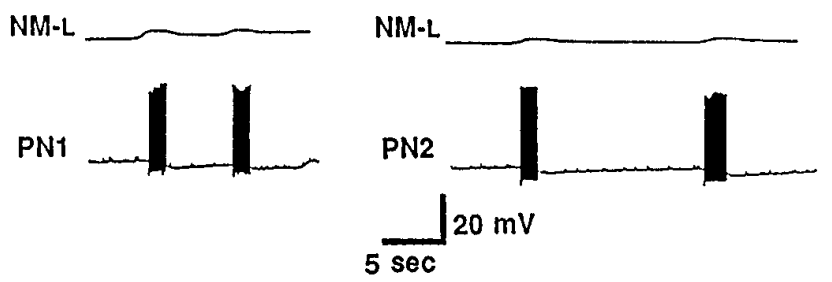

Figure 8. Effects of CC5 on putative neck motor neurons. A, Schematic drawing shows cell body locations and axon distributions of two neurons that receive monosynaptic connections and were labeled by carboxyfluorescein dye in the same preparation $(n=5)$. $A 1$, Pedal neuron 1 (PN1). $A 2$, Pedal neuron 2 (PN2). These cells appear to be members of a small class of neurons with similar or identical properties. The ganglia are illustrated dorsal side up, with the pleural-abdominal connective directed out of the pleural ganglion at the 11 o'clock position. $B$, CC5 evoked short-latency EPSPs in both the PN1 $(B 1 a)$ and PN2 (B1b) neurons. The EPSPs in the PN1 and PN2 neurons were not blocked by a high-divalent cation solution $(B 2 a, B 2 b)$, suggesting that CC5 may make monosynaptic connections to these neurons $(n=5)$. $C$, Isotonic strain gauge records of longitudinal contractions evoked in neck muscles by firing of PN1 $(C 1)$ or PN2 $(C 2)(n=5)$. The preparation was identical to that described in Figure 6.
A
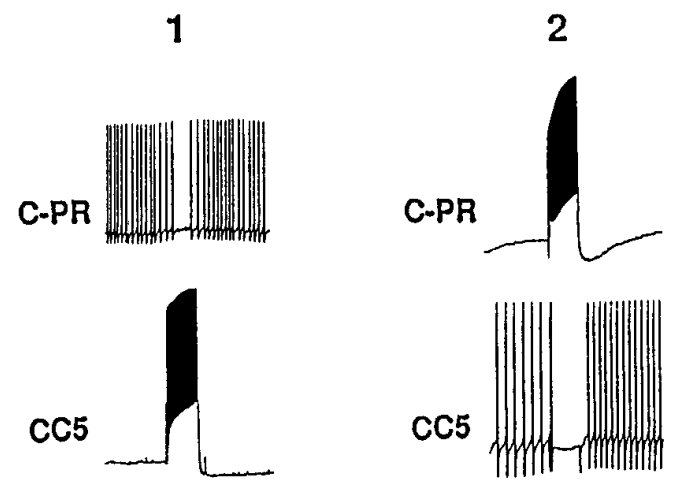

B

1
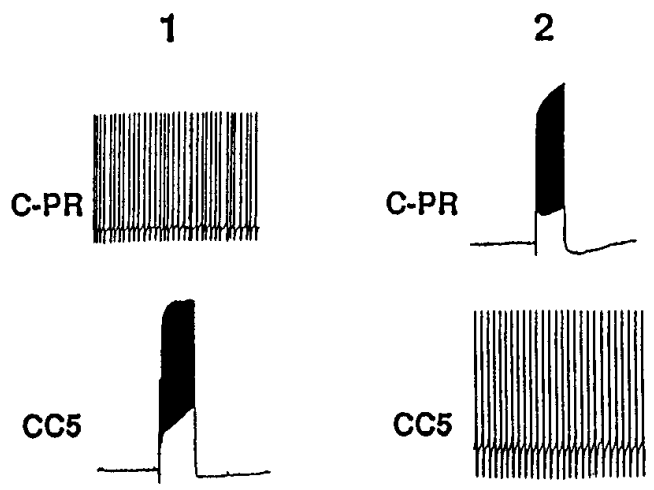

$\operatorname{cc5}$

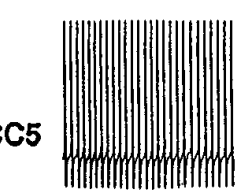

$20 \mathrm{mV}$

Figure 9. Mutual interaction between CC5 and C-PR. $A, \mathrm{CC} 5(A 1)$ and C-PR $(A 2)$ mutually inhibit each other. The cells are fired at $\sim 15 \mathrm{~Hz} . B 1$, $B 2$, The inhibitory actions are not evident in a solution containing highdivalent cations $(n=6)$.

\section{Effects of firing of CC5 on the activity of neurons in the cerebral ganglion}

\section{Feeding-related neuron $C-P R$}

Neuron C-PR (cerebral-pedal regulator) is located bilaterally in the cerebral $\mathrm{M}$ cluster. C-PR is activated when the animal is aroused by food (Teyke et al., 1990b, 1991). We found that firing $\mathrm{CC} 5$ at a high rate $(>15 \mathrm{~Hz})$ inhibited the activity of C-PR (Fig. 9A1). Similarly, firing of C-PR inhibited CC5 (Fig. 9A2). The inhibition of either cell was not associated with the presence of distinct fast IPSPs. The inhibitory effects of the neurons on one another were reversibly blocked when the head ganglia were bathed in a high-divalent cation solution (Fig. 9B1,B2). Although this experiment suggests that the connections between the two cells involves an interposed interneuron, the experiment is not definitive because the inhibitory effects required relatively high frequency of firing of the cells, and it was not possible to precisely match this frequency in the high-divalent cation solution.

The inhibition that C-PR evokes in CC5 suggests that C-PR activity should be associated with a decrease of activity of PAS, the excitatory follower of CC5. Indeed, we found that firing of C-PR bilaterally inhibited firing of PAS (Fig. 10A). The inhibition of PAS could be an indirect effect attributable to the inhibition of CC5 (which excites PAS), but we also found that C-PR appeared 
A

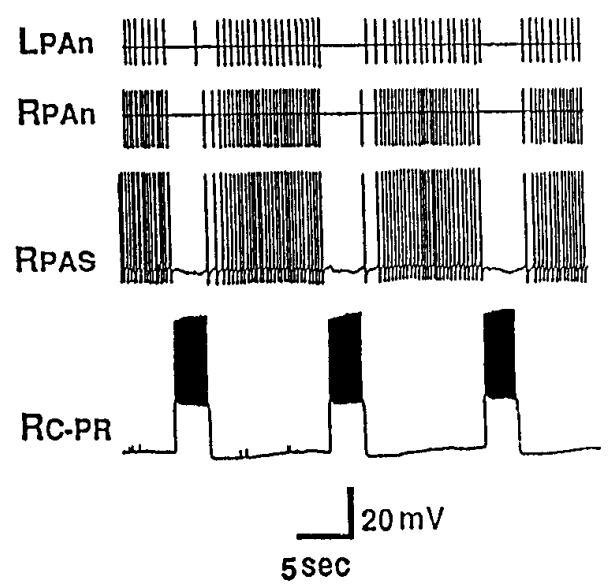

B
1

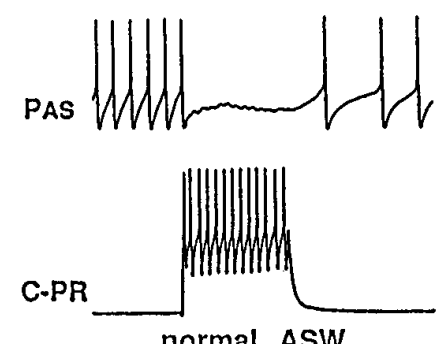

normal ASW
2

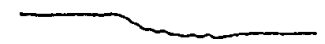

$\mathrm{HI} \mathrm{Mg++}(3 \mathrm{X}), \mathrm{HI} \mathrm{Ca++(3X)}$

$20 \mathrm{mV}$
Figure 10. Effects of the excitatory follower of CC5, C-PR, on PAS. $A$, $\mathrm{C}-\mathrm{PR}$ sends an axon from the cerebral ganglion to the ipsilateral and contralateral pedal ganglia via the cerebral-pedal connective, and firing of C-PR could inhibit both the ipsilateral and contralateral PAS neurons. Traces show intracellular recording from the ipsilateral PAS and extracellular recordings from the ipsilateral and contralateral PAS recorded in the left and right pedal artery nerves $(L P A n, R P A n)$. $B$, The inhibition of PAS produced by firing of C-PR $(B 1)$ was not blocked in a high-divalent cation solution $(B 2)$, indicating that C-PR may make a monosynaptic connection to $\operatorname{PAS}(n=8)$.

to directly evoke an IPSP in PAS (Fig. 10B1), and the IPSP persisted in a high-divalent cation solution (Fig. 10B2). This inhibitory connection accounts, at least in part, for the previously described inhibition of PAS produced by electrical stimulation of the pedal connective (Xin et al., 1996). The mutual inhibitory interrelation between $\mathrm{CC} 5$ and C-PR is consistent with a role of CC5 in contributing to neck shortening, because when animals are aroused by food, C-PR contributes to the generation of a head lifting response in which the neck of the animal extends and lengthens (Teyke et al., 1990b).

\section{Metacerebral cell}

We found that CC5 had polysynaptic effects on the metacerebral cell (MCC), a serotonergic neuron that is active when the animal is in a food-aroused state and that produces a number of the behavioral effects associated with food-induced arousal (Weiss et al., 1978; Kupfermann and Weiss, 1982; Rosen et al., 1989). When CC5 was fired, MCC firing was briefly suppressed and was then enhanced (Fig. 11A). To reveal any underlying synaptic potentials, spike activity of the MCC was suppressed by hyperpolarizing current (Fig. 11B). The hyperpolarized MCC typically exhibited a spontaneous, regular depolarizing potential, which probably corresponds to the compound excitatory-inhibitory potential (the inhibitory component is flipped) that can be observed when the cell is at resting potential and is firing (Weiss and Kupfermann, 1976). Firing of CC5 evoked a brief burst of depolarizations, which appear to represent an increase of the firing of the interneuron that produces the excitatory-inhibitory potentials in the MCC. The brief burst of the depolarizing potential abruptly terminated, followed by a period in which the spontaneous depolarizing potential was no longer evident, suggesting that the relevant interneuron ceased to fire. Thus, it appears that the effects of CC5 on the MCC are attributable to an initial increase of inhibition produced by one or more interneurons, followed by disinhibition and perhaps postinhibitory rebound.

\section{Histaminergic neuron $\mathrm{C} 2$ and other neurons in the cerebral E cluster}

The MCC receives excitatory input from the histaminergic mechanosensory neuron C2 (Ono and McCaman, 1980; McCaman and Weinreich, 1985; Weiss et al., 1986). We therefore examined the interrelation between $\mathrm{CC} 5$ and $\mathrm{C} 2$. The $\mathrm{C} 2$ neuron was identified by its position in the $\mathrm{E}$ cluster and its characteristic excitatory effect on the MCC (Weiss et al., 1986) (Fig. 12 A1) and on E cluster neurons C4 or C5 (Chiel et al., 1986). After a long latency, C2 was inhibited when CC5 was fired (Fig. 12A2). CC5 also was found to inhibit various other E cluster neurons (Fig. 12B,C) that receive excitatory or inhibitory synaptic inputs from $\mathrm{C} 2$. Some $\mathrm{E}$ cluster neurons appeared to be inhibited after a relatively long delay (1-2 sec) when CC5 was fired, and prolonged firing of CC5 could alter a tonic firing pattern to a burst pattern in these neurons. Figure 13 shows an example of this type of effect on $\mathrm{E}$ cluster neuron C4 (identified by criteria outlined in Chiel et al., 1986) when CC5 was tonically fired at $\sim 4-5 \mathrm{~Hz}$ in a preparation consisting of the cerebral- and pedal-pleural ganglia without the buccal ganglion. Simultaneous recordings from the cerebral end of a $\mathrm{CBC}$ in such preparations revealed that the bursts of synaptic input to $\mathrm{C} 4$ were coincident with bursts of firing of one or more neurons that send an axon into the connective (Fig. 13, top trace). No obvious synaptic input was apparent in CC5 during these bursts.

\section{Relationship of CC5 firing to the activity of the buccal mass and command-like neuron $\mathrm{CBI}-2$}

Besides the connections to C-PR and the MCC, CC5 excited or inhibited many neurons in the cerebral ganglion, including a number of previously identified cells. In every case examined, the synaptic effects were not direct, but may have been mediated by interneurons located in the pedal or pleural ganglion, because the synaptic effects were eliminated by cutting the C-PL connectives. One such neuron affected by CC5 was cerebral-to-buccal interneuron 2 (CBI-2), a cell that drives a buccal motor program (Rosen et al., 1991). Simultaneous recording from CC5 and CBI-2 is technically difficult, because CBI-2 is located on the ventral surface of the cerebral ganglion, whereas CC5 is on the dorsal surface. Therefore, the cerebral ganglion was pinned dorsal surface up; CC5 was impaled, and then CBI-2 was impaled blind by advancing the electrode through the ganglion in the vicinity of the soma of CBI-2. If the buccal ganglion was disconnected from the cerebral ganglion, firing of CBI-2 had no obvious effects on the activity of CC5. In experiments in which the buccal 
Figure 11. Effect of $\mathrm{CC} 5$ on the serotonergic modulatory neuron MCC. $A$, Firing CC5 produced inhibitory-excitatory synaptic potentials in the MCC. $B$, Hyperpolarizing the MCC while firing CC5 revealed that CC5 evoked a burst of synaptic potentials (presumably attributable to activation of an interneuron) that appeared to be reversed IPSPs. Termination of the IPSPs occurs at the time that the MCC is excited. Thus, the excitation may be the result of postinhibitory rebound $(n=5)$.
A

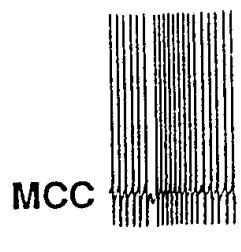

B

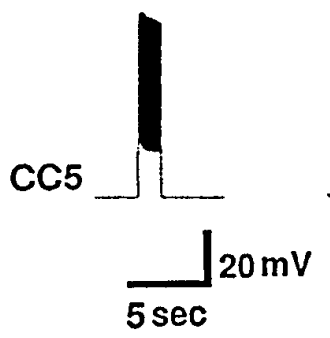

ganglion was left connected to the cerebral ganglion, as found previously (Rosen et al., 1991), injection of constant depolarizing current into CBI-2 evoked a buccal motor program that was associated with a rhythmic excitation and inhibition of the firing of CBI-2 and of other cerebral cells such as C4. Firing of a burst of spikes in CC5 interrupted the buccal program evoked by CBI-2

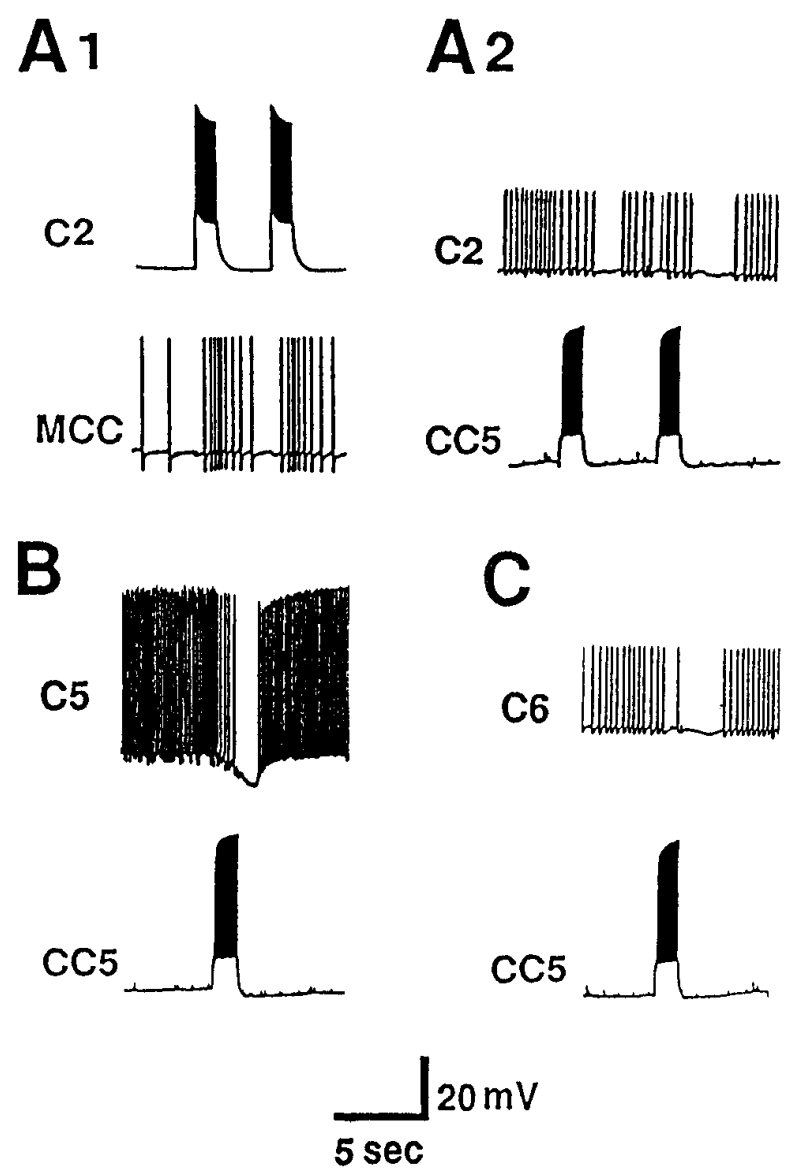

Figure 12. Effect of $\mathrm{CC} 5$ on neurons in the E cluster. The histaminergic mechanoafferent $\mathrm{C} 2$ was identified by its position and its excitatory effect on the MCC (A1). A2, The $\mathrm{C} 2$ neuron could be inhibited by firing of CC5. $B$, CC5 also inhibited the E cluster neurons $\mathrm{C} 5$ and $\mathrm{C} 6$, which were identified based on their position and their input from C2 (Chiel et al., 1986) $(n=8)$. and terminated the rhythmic synaptic inhibition (Fig. 14). This effect of CC5 appeared to be dependent on the C-PL connectives and CBCs. At least some of the rhythmic synaptic inputs to CBI-2 appear to be attributable to the firing of interneurons that are located in the buccal ganglion (Chiel et al., 1988; Rosen et al., 1991) (our unpublished observations). Because CC5 does not send an axon to the buccal ganglion, our observations suggest that the effects of CC5 on CBI-2 may involve the activation of interneurons located in the pedal-pleural ganglia that have direct or indirect effects on neurons in the buccal ganglia.

\section{Effects of CC5 on other cerebral ganglion neurons}

Firing of CC5 produced polysynaptic effects on a number of neurons in the $\mathrm{M}$ cluster. Two neurons were found to be inhibited (Fig. 15A,B). Based on their size and position, these are identified as cells $\mathrm{C} 11$ and $\mathrm{C} 12$, neurons that send axons into the lower labial nerve and produce movements of the perioral region (S. Rosen, unpublished observations). Two other neurons in the $\mathrm{M}$ cluster that have not been identified previously, were found to receive polysynaptic excitatory inputs from the ipsilateral CC5 (Fig. $15 C, D)$. Intracellular dye fills of these neurons, which we term $\mathrm{C} 13$ and $\mathrm{C} 14$, revealed that one (C13) sends an axon into the upper labial nerve, whereas the other (C14) sends an axon into the anterior tentacle nerve. Firing of these neurons produced movements of the medial part of the anterior tentacle, suggesting that they may be motor neurons.

\section{Effects of CC5 on neurons in the abdominal ganglion Neuron R15}

Neuron R15 was one of several identified neurons in the abdominal ganglion whose activity was altered when CC5 was fired. In some preparations, the activity of R15 could be monitored via extracellular recording of its small branch that travels in the left PAn (Skelton and Koester, 1992). When R15 was spontaneously bursting at a regular rate, firing of $\mathrm{CC} 5$ decreased the interburst interval (Fig. 16A). Intracellular recording from R15 revealed spontaneous excitatory input from a single interneuron, perhaps interneuron XIII, which appears to be located in the pedal ganglion (Segal and Koester, 1982). Firing of CC5 increased the frequency of this EPSP, and no other EPSPs appeared to be recruited (Fig. 16B).

Because it has been suggested that R15 may be involved in regulating egg-laying behavior and that $\mathrm{CC} 5$ could be involved in head turning movements during egg-laying behavior, we tested 


\section{C-BC}
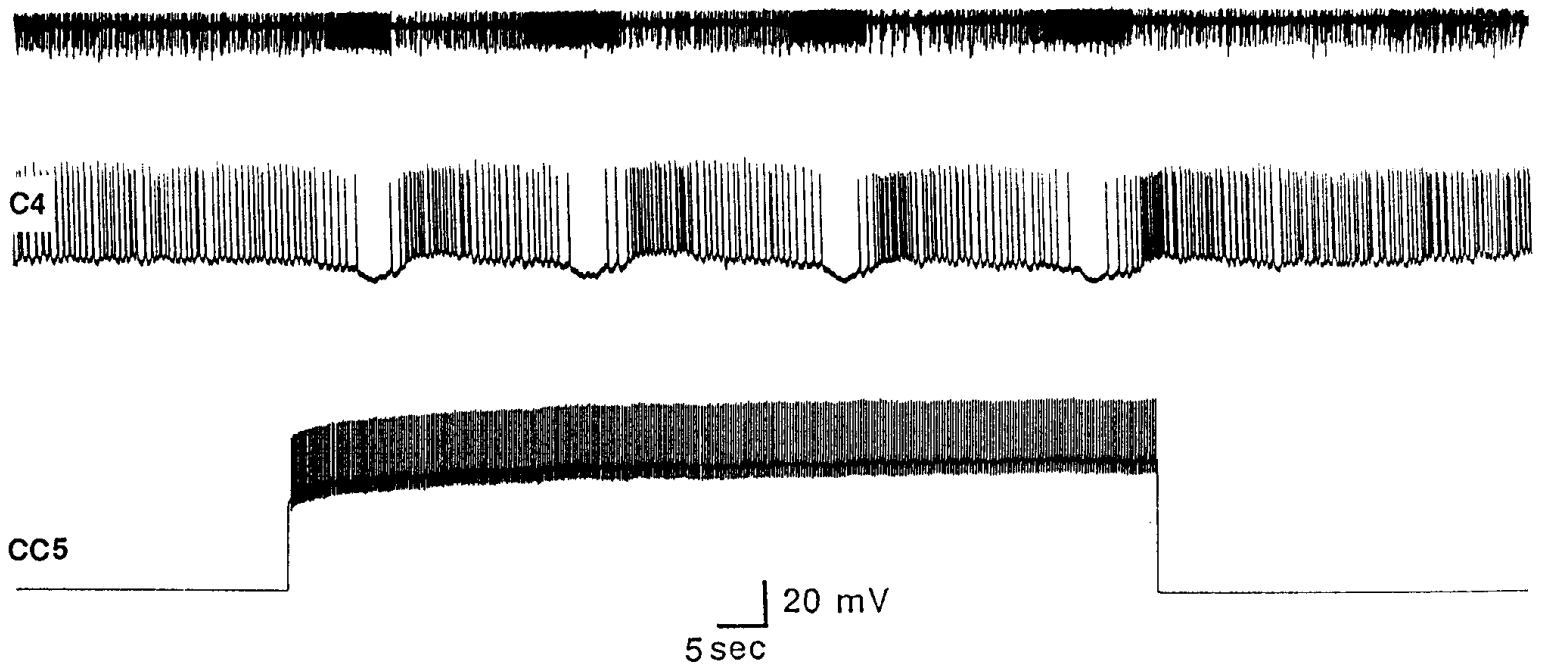

Figure 13. Inhibition of the $\mathrm{C} 4$ neuron by $\mathrm{CC} 5$ could occur with a long delay, and prolonged firing of CC5 could induce rhythmic firing in $\mathrm{C} 4$ concomitantly with burst of firing of one or more units that send axons to the $\mathrm{CBC}$ (Recording is from the cerebral end of the cut $\mathrm{CBC}$. The buccal ganglia are not present.) $(n=6)$.

the effect of bag-cell extracts, which elicit egg-laying behavior in the intact animal (Kupfermann, 1970; Ferguson et al., 1989). The results from four preparations failed to show significant changes in the activity of CC5 when the bag-cell extract was added to the artificial sea water (ASW).

\section{Cardiovascular neurons}

The LB vasoconstrictor ( $\mathrm{LBvc}$ ) neurons of the abdominal ganglion were previously demonstrated to be involved in alteration of blood flow during buccal mass movements that occur during the consummatory phase of feeding (Koch et al., 1984). They fire in phase with rhythmic biting and route blood flow alternatively to the head (during protraction of the buccal mass) and to the digestive system (during retraction). Because the activity of CC5 appears to be correlated with buccal mass movements, we examined its effects on LBvc neurons. Some LBvc neurons were found to receive polysynaptic excitatory inputs when CC5 was fired (Fig. $17 A$ ). The resulting excitation of LBvc neurons did not appear to be sufficiently strong to evoke a visible contraction in either the

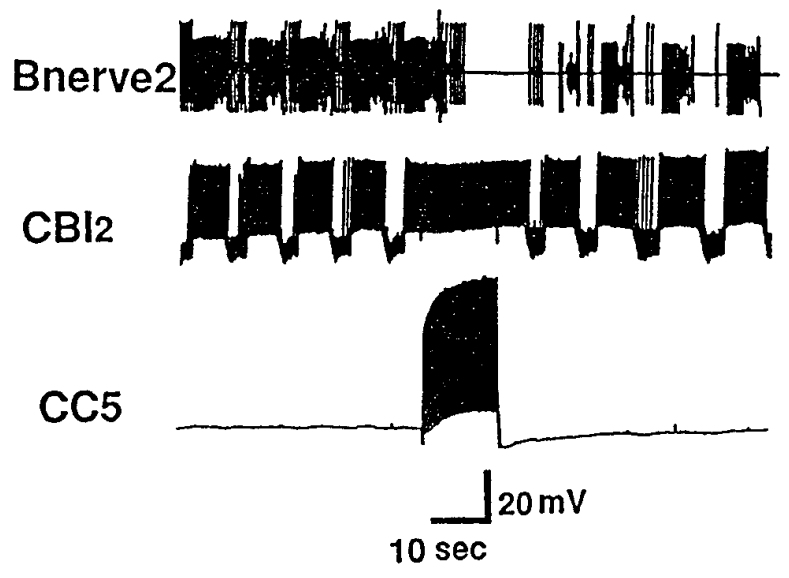

Figure 14. A buccal motor program was induced by tonic depolarization of CBI-2. Firing of CC5 interrupted the rhythmic firing of CBI-2 by inhibiting an interneuron that produces rhythmic synaptic input to CBI-2 $(n=5)$. abdominal or gastroesophageal artery in preparations of isolated ganglia with the heart and blood vessels attached.

Firing of CC5 also produced a clear excitatory effect on the $\mathrm{RB}_{\mathrm{HE}}$ cell (Fig. 17B), a neuron that excites the heart. There were
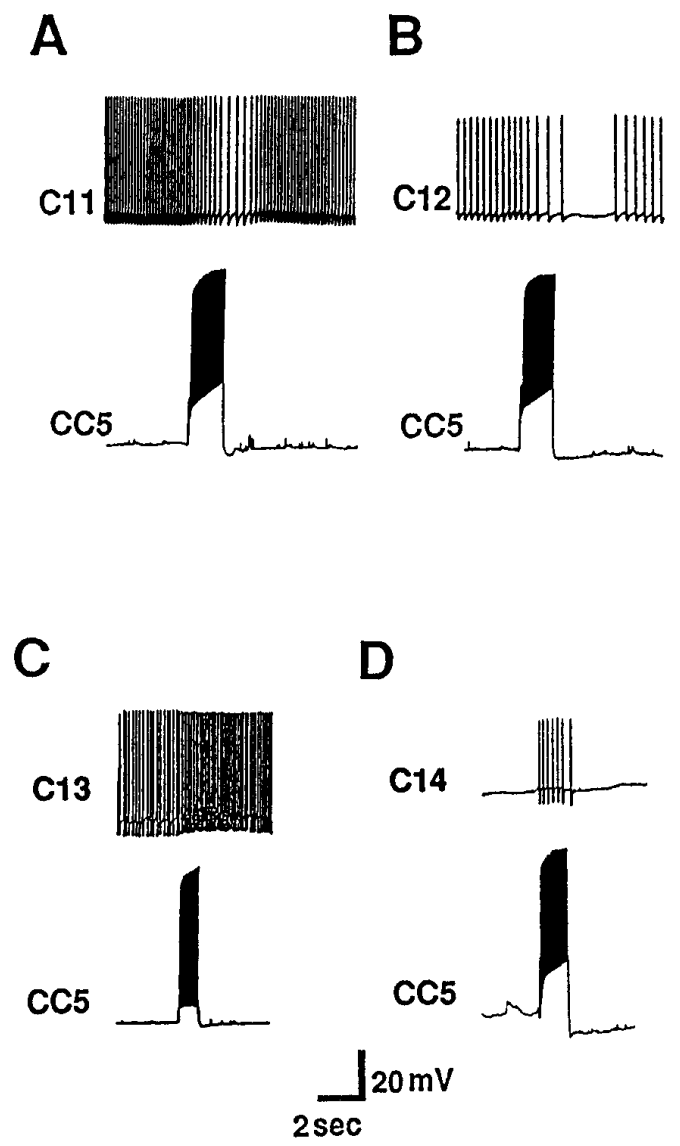

Figure 15. Inhibitory effect of CC5 on identified cerebral neurons $\mathrm{C} 11$ $(A)$ and $\mathrm{C} 12(B) . C, D$, Two other neurons in the M cluster (C13 and C14) were excited by CC5 $(n=7)$. 
A
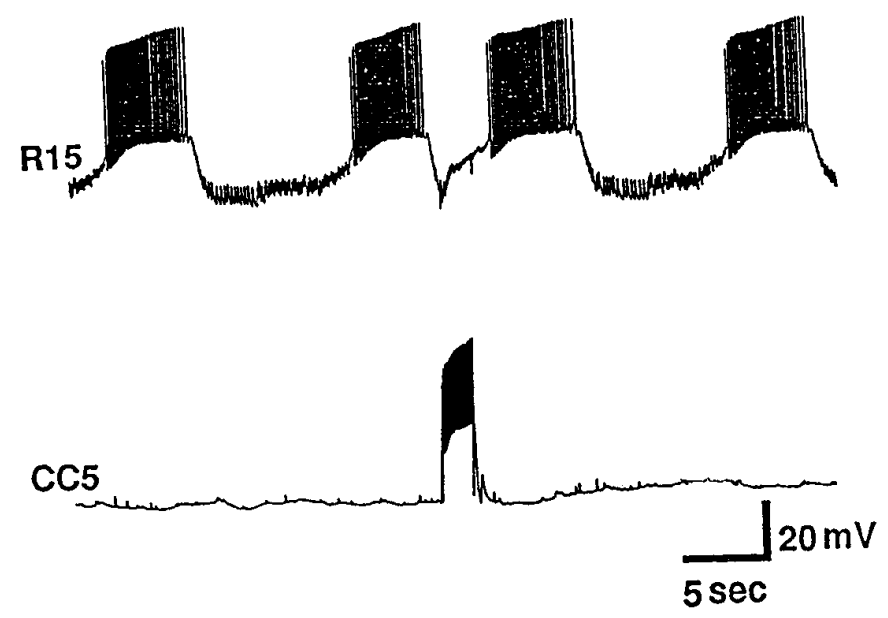

B

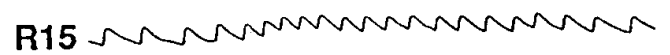

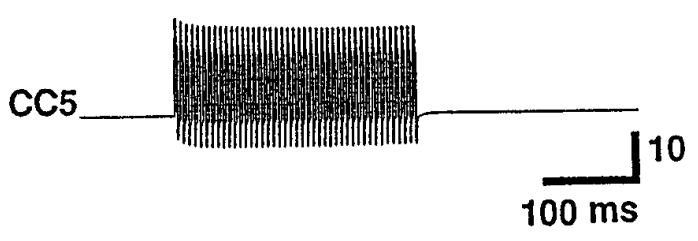

Figure 16. Effect of $\mathrm{CC} 5$ on abdominal ganglion neuron R15 recorded intracellularly. $A$, Firing of the right $\mathrm{CC} 5(15 \mathrm{~Hz})$ excited R15 and changed its rhythmic firing pattern. $B$, Fast trace recording from R15 revealed that the CC5 increases the firing of an excitatory interneuron $(n=8)$.

several other unidentified abdominal ganglion neurons that received inputs when CC5 was fired. Because CC5 does not send an axon to the abdominal ganglion, the effects of CC5 on these neurons may be mediated by interneurons in the circumesophageal ganglia.

\section{In vivo recording of PAS before and after cutting of its connection with CC5}

Based on the diverse inputs and outputs of CC5, we predicted that it would be active during many different behaviors. Because we could not record from CC5 in intact animals, we instead recorded from the peripheral axon of its monosynaptic follower, PAS. Skelton and Koester (1992) had already reported that PAS is active during several behaviors. We confirmed the previous findings that the PAS fires when the animal spontaneously turns its head toward the ipsilateral side (Fig. 18A) and the neuron is inhibited during contralateral turns. Our previous data are consistent with the idea that CC5 activity is related to behaviors involving shortening of the neck. Thus, its activity during head turns might be more closely related to neck shortening rather than to neck turns per se. Moderate head turns can occur in which the

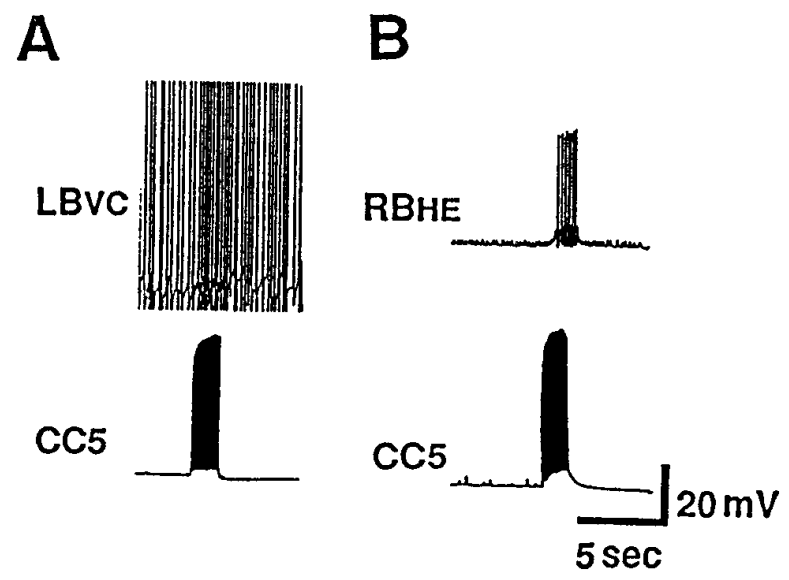

Figure 17. Effect of CC5 on abdominal ganglion neurons involved in the cardiovascular system. $A$, CC5 polysynaptically excites vasoconstrictor neuron $\mathrm{LBvc}$, and heart motor neuron $\mathrm{RB}_{\mathrm{HE}}(B)(n=8)$. center of rotation of the neck is at the base of the neck, and the neck itself does not appear to shorten (Kuenzi and Carew, 1994b) (compare positions 2 and 3 with position 4 in Fig. 18Ac). We observed that relatively small head turns, during which the neck did not substantially shorten, could indeed occur with little or no excitation of CC5 (Fig. 18Aa2,Aa3). Substantial firing (or contralateral inhibition) of CC5 occurred only when there was a relatively large turn in which the ipsilateral side of the neck shortens (Fig. 18Aa4,Aa1). We also observed that during the head extension that occurs during the initial exposure to food, PAS activity is suppressed (Fig. 18Ba).

Firing of the PAS also rhythmically increased during biting and swallowing movements, in phase with backward rotation and retraction of the radula (Fig. 18Ca). As reported previously (Skelton and Koester, 1992), PAS also fired phasically during locomotion (spontaneous or evoked by a salt stimulation to the tail) at the step phase when the tail is released and the neck shortens (Fig. 18Da).

To further examine the possible function of CC5, we studied the activity of PAS in animals in which a CC5 neuron was disconnected from the pedal-pleural ganglia. This was accomplished by sectioning of the C-PL connective, the only direct route of CC5 out of the cerebral ganglion. Any activity of the ipsilateral PAS that is directly driven by activity of CC5 should be eliminated by this lesion, because the only axon of CC5 out of the cerebral ganglion is in the C-PL connective, and CC5 has a strictly unilateral effect on PAS (Xin et al., 1996). We reported previously that this lesion completely eliminates the response of PAS to tactile stimuli applied to the head (Xin et al., 1996). The current results indicate that after section of the C-PL connective, firing of the PAS also no longer occurred during head turns (Fig. 18Ab). For biting behavior, after section of the pleural connective, modulation of PAS was reduced, but did not appear to be completely eliminated (Fig. 18Cb). Strikingly, the PAS continued to fire strongly during the neck shortening phase of locomotor behavior (Fig. 18Db). Because studies in the isolated nervous system indicate that CC5 activity is modulated in phase with locomotor or feeding programs, it appears that even though the locomotor and feeding circuitry have access to PAS via CC5, there is access to PAS via alternative parallel pathways. Inhibition of PAS firing 
A

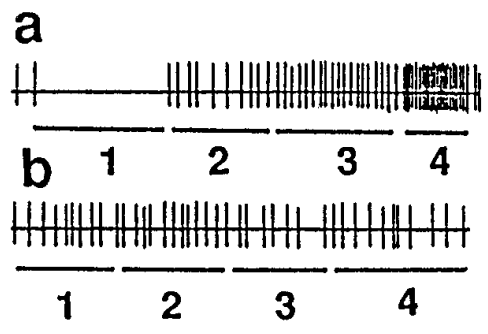

C
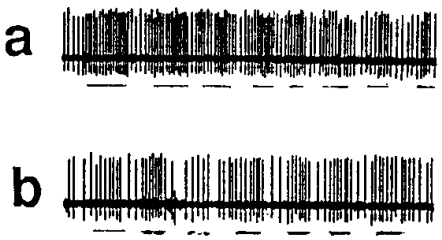

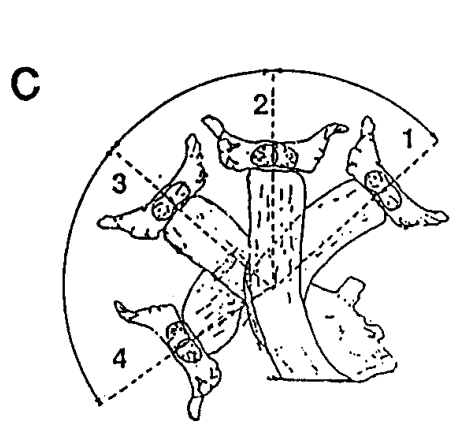

B

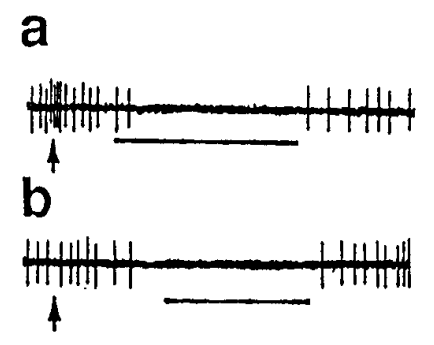

\section{D}

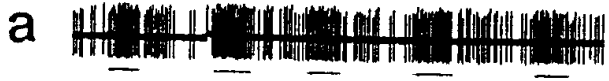

b

\section{$5 \mathrm{sec}$}

Figure 18. Firing of PAS recorded in the PAn during different behaviors before and after cutting of the ipsilateral C-PL connective $(n=8)$. $A$, Recordings during head turns elicited by contact of food to the tentacles. $A a$, Recording before the lesion of the C-PL connective. Strong firing of PAS occurred only with the largest turn (position 4). Ab, Recording after the lesion of the C-PL connective. Firing of PAS was not affected by the position of the head. $A c$, Line drawing of the position of the head during various turns. The numbers correspond to the numbers indicated in parts $A a$ and $A b$. $B$, Inhibition of PAS during head lifting (line) before $(a)$ and after $(b)$ cutting of the ipsilateral C-PL connective. $C$, Modulation of PAS activity during spontaneous buccal mass movements (backward movement indicated by broken lines) before $(a)$ and after $(b)$ cutting of the ipsilateral C-PL connective. $D$, Modulation of PAS activity during spontaneous stepping movements (neck shortening phase of step indicated by broken lines) before $(a)$ and after $(b)$ cutting of the ipsilateral C-PL connective.

during head lifting persisted after section of the C-PL connective (Fig. 18Bb). This finding is consistent with the observations that head lifting involves activity of C-PR, and C-PR can inhibit PAS via the C-PR axon that travels in the cerebral-pedal connective.

Firing of CC5 evokes unit activity in the $\mathrm{CBC}$ and can alter burst activity recorded in buccal nerves. Because strong noxious stimuli applied to the head produce strong activation of CC5 (Xin et al., 1996), we examined the effect of noxious stimuli on buccal ganglion activity recorded from buccal nerve 2 in free-moving animals. Rhythmic activity of the buccal mass and of buccal nerve 2 was evoked by applying seaweed to the lips of the animal. Because noxious stimuli cause the animal to withdraw from the food, we presented the noxious stimuli after the food was removed and the buccal mass exhibited bouts of "spontaneous" burst activity. This spontaneous burst activity was transiently inhibited by a pinch to a tentacle (Fig. 19A). Rhythmic activity evoked during swallowing behavior that occurs during the ingestion of a strip of food was not inhibited by a pinch to a tentacle (Fig. 19B). In animals in which the C-PL connectives were severed and which recovered feeding behavior, a pinch was no longer effective in suppressing the spontaneous rhythmic bursting in buccal nerve 2 (Fig. 19C).

\section{DISCUSSION}

We previously provided evidence that CC5 or the pair of CC5 neurons may be critical interneurons in the circuitry that controls the arterial shortening, which occurs during withdrawal responses of the head and tentacles (Xin et al., 1996). The present paper provides both indirect and direct evidence suggesting that CC5 is a multifunctional interneuron that serves different roles in a very wide variety of behaviors. Our experiments specifically support the hypothesis that CC5 is involved in at least six different behaviors. We suggest that each of these behaviors, which superficially do not appear to be all related to one another, may have in common a component involving changes in the length of the neck. CC5 appears to be involved in orchestrating a coordinated response that engages both the somatic as well as the visceral musculature of the animal. Although CC5 may mediate a specific behavioral response, the precise functional role played by CC5 for each of the six behaviors in which it is engaged, appears to be different. The suggested role of CC5 in different behaviors is not unequivocally demonstrated by any single experiment, but rather is supported by converging lines of evidence.

The analysis of the function of individual neurons in behavior often does not lend itself to simple labels or brief verbal descriptions. In this discussion, we have attempted to provide a commonsense feel for the multifunctional operation of CC5 by using two types of analyses. First, we describe our proposed characterization of the various roles of functioning of CC5 for each behavior in which it appears to be involved based on the following functional criteria: (1) whether the two cells fire concurrently or not; (2) 
Table 1. Roles of CC5 function in six behaviors

\begin{tabular}{|c|c|c|c|c|c|c|}
\hline \multirow[b]{2}{*}{ Mode } & \multicolumn{6}{|l|}{ Behavior } \\
\hline & Locomotion & Head turn & Head lift & $\begin{array}{l}\text { Head } \\
\text { withdrawal }\end{array}$ & $\begin{array}{l}\text { Local } \\
\text { withdrawal }\end{array}$ & Feeding \\
\hline Unilateral & & $X$ & & & $\mathrm{X}$ & \\
\hline Reflex & & & & $\mathrm{X}$ & $\mathrm{X}$ & \\
\hline $\mathrm{CPG}$ & & & & & & $\mathrm{X}$ \\
\hline Command & & $\mathrm{X}$ & & & $\mathrm{X}$ & \\
\hline Command system & & & $\mathrm{X}$ & $\mathrm{X}$ & & $\mathrm{X}$ \\
\hline Contributory & $\mathrm{X}$ & & & & & \\
\hline
\end{tabular}

whether the cells are activated reflexly or via an organized, endogenously active central pattern generator; and (3) whether the cells are necessary and sufficient for behaviors or components of behaviors, specifically the pedal artery shortening component of the various behaviors. In a second type of analysis, we describe the function of the two CC5 neurons in terms of the type of combinatorial modes they appear to use during different behaviors, and briefly discuss the role of CC5 for each behavior.

\section{Functional roles of CC5}

As indicated in the first functional category in Table 1, CC5 can function in a unilateral or a bilateral mode. For example (Xin et al., 1996), during local withdrawal elicited by a weak stimulus applied to one tentacle, only the ipsilateral cell is active, whereas during head withdrawal elicited by a strong stimulus to a tentacle,
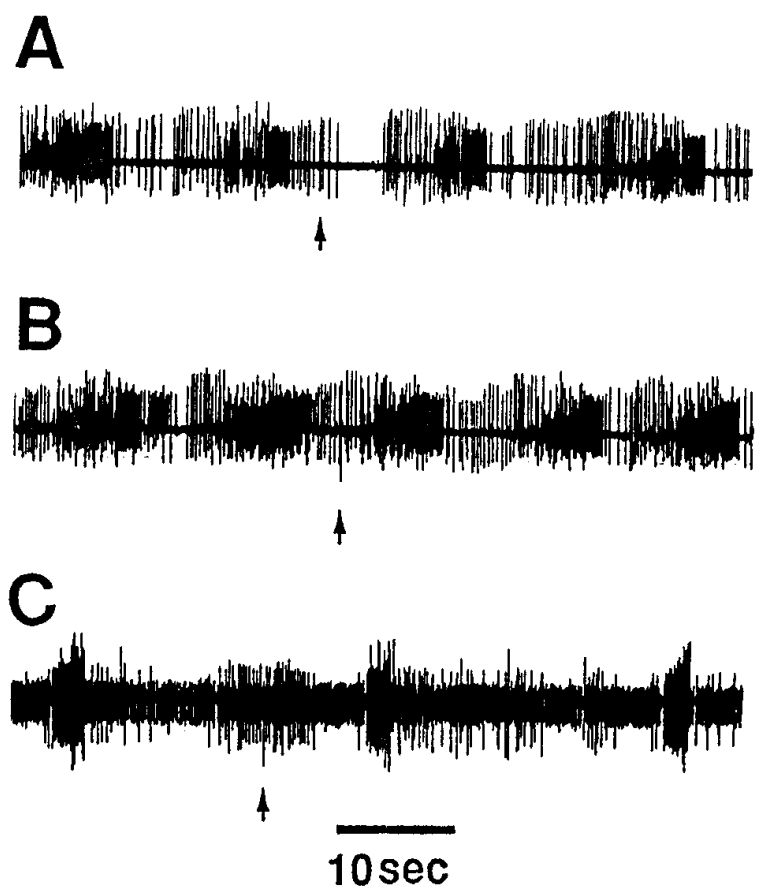

Figure 19. Effect of pinching a tentacle on buccal ganglion activity recorded in buccal nerve 2 in free-moving animals $(n=6)$. $A$, During spontaneous biting movements, a pinch to an anterior tentacle produced a brief inhibition of unit activity in nerve 2 . Nerve pinch was not effective in inhibiting activity during swallows, induced by a strip of food $(B)$, or during spontaneous biting movements after severance of the C-PL connectives $(C)$. both the ipsilateral and contralateral CC5 neurons are active. Conversely, during head lifting, when the neck extends, both CC5 cells appear to be inhibited, and because they are normally active, their inhibition can contribute to a relaxation response of the pedal artery. It is important to realize that the unilateral versus bilateral mode of functioning of CC5 results in CC5 contributing to qualitatively different responses, i.e., head and neck turning versus head withdrawal.

A second class of function of CC5 relates to the source of the input that activates it. In some behaviors, it receives endogenous input from presumptive central pattern-generating neurons; for other behaviors, it appears to be driven reflexly. Furthermore, for some behaviors it appears that it can be driven either reflexly or by a central pattern generator.

The third class of function of CC5 relates to the issue of the degree to which individual CC5 cells control behavior or given components of behavior. We have specifically examined this question with regard to the role of CC5 in controlling the shortening of the pedal artery. This response appears to be a component of multiple behaviors. For the pedal artery shortening component of different behaviors, CC5 cells can function in a command-like manner: they can function as a bilateral command system (Kupfermann and Weiss, 1978) or they can function together in a contributory manner with other neurons that are activated in parallel.

\section{Combinatorial codes during multiple behaviors in which CC5 appears to be active}

The functioning of the two CC5 neurons illustrates how an individual neuron may be engaged in different behaviors and code for different information as a function of the concurrent activity of other neurons. In a simplified manner, for a given behavior a CC5 neuron can be thought of as exhibiting either an increase, decrease, or no change in its baseline activity. Because there are two CC5 neurons, the cells can, in principle, code for nine major modes of function. As indicated in Table 2, we have found that the cells appear to use seven of the nine modes of function.

All of these behaviors in which CC5 appears to be involved seem to involve alterations of the length of the neck. We distinguish at least six variations of neck movements. (1) During locomotion, the neck shortens bilaterally during each step, after detachment of the tail from the substrate. (2) During head turning, the neck shortens on the side of the turn, but only at the extremes of horizontal movement. (3) During defensive withdrawal in response to a strong noxious stimulus, the neck exhibits a strong bilateral shortening. (4) During local retraction elicited by a 
Table 2. Behavioral function associated with increase, decrease, or no change of left or right $\mathrm{CC} 5$ neuron activity

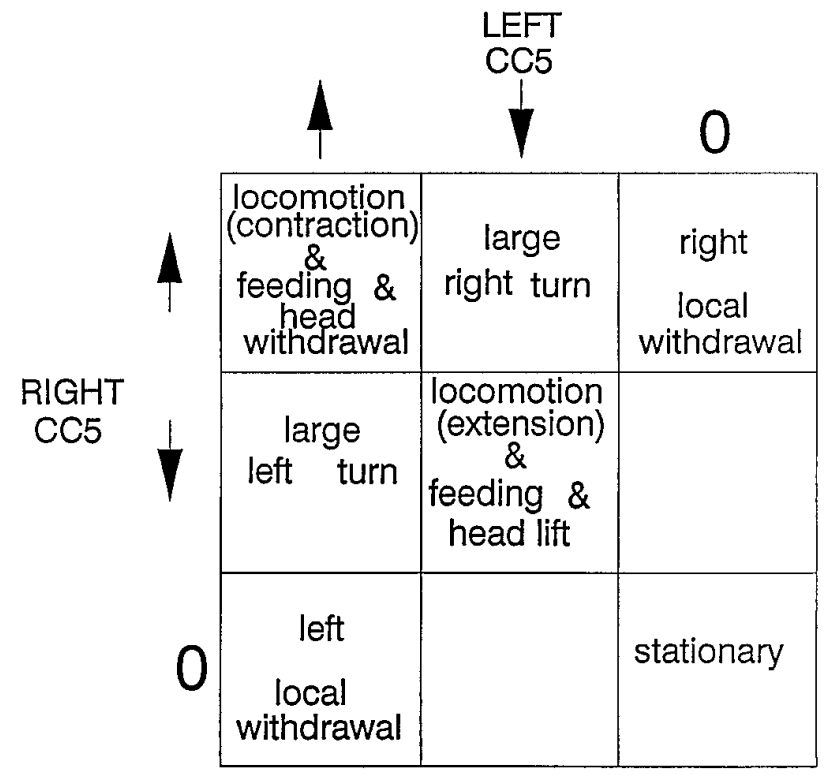

Up arrow, Increase of cell firing; down arrow, decrease of cell firing; 0 , no change of cell firing. Contraction and extension refer to the phases of locomotion in which the head and neck contract or extend.

relatively weak tactile stimulus, the neck shows a small ipsilateral shortening. (5) During the appetitive phase of feeding behavior, the neck extends when the animal becomes food-aroused and lifts its head. (6) During the consummatory phase of feeding behavior, there may be small rhythmic movements of the neck during repetitive biting movements. Neck movements also occur during reproductive mating behavior (Susswein et al., 1984; Ferguson et al., 1989; Bernheim and Mayeri, 1995) and phototactic responses (Kuenzi and Carew, 1991), but we have not examined the role of CC5 during these responses.

\section{Withdrawal responses}

Our previous paper (Xin et al., 1996) provided evidence that CC5 is involved in a withdrawal response to a weak unilateral tactile stimulus applied to the head. An individual CC5 appears to be necessary and sufficient for the pedal artery contraction component of the multicomponent withdrawal response to a weak stimulus, whereas for a stronger stimulus the two cells are active as a command system for the bilateral arterial response component.

\section{Head turning}

Based on recordings from PAS in free-moving animals, it appears that CC5 significantly contributes to head turns only when the turns are near maximal and there is substantial shortening of the neck. In this case, the ipsilateral cell is active, and because the response of PAS appears to be eliminated by cutting the C-PL connective (which disconnects CC5 from the PAS), it is possible that $\mathrm{CC} 5$ is necessary and sufficient for arterial shortening for extreme head turns. Although CC5 has clear asymmetric effects on neurons that cause contractions of neck muscles, the specific role of CC5 in controlling neck muscles during head turns is obscure. Firing of CC5 was shown to drive neck motor neurons at a rate sufficient to produce robust contraction of neck muscles. Nevertheless, elsewhere (Xin and Kupfermann, 1995) we report that head turns appear normal after a unilateral section of the pleural connective, the pathway that provides the only direct route by which CC5 can affect the pedal and pleural motor system that controls head turns. It was found, however, that although the unilateral lesion did not have a detectable effect on head turns, it exacerbated the deleterious effects of sectioning of a pedal connective. Thus, it is possible that the effects of CC5 on head turning are relatively subtle and are manifest only when combined with the activity of other cerebral neurons, the axons of which pass through the pedal connective.

\section{Locomotion}

In contrast to the situation for extreme head turns, and for head withdrawal, firing of PAS still occurs during locomotion even when the ipsilateral C-PL connective is severed. Because CC5 provides excitatory input to PAS and because CC5 appears to be active during presumptive fictive locomotion in reduced preparations, it is likely that its activity contributes to PAS firing during locomotion, but during the locomotor program there is clearly a strong alternative input to PAS.

\section{Feeding}

CC5 receives input associated with bursting of the buccal ganglion, and the PAS neurons are active during feeding movements. Observation of animals indicates that there are only slight movements of the neck during bite and swallows. Thus, it is possible that during feeding behavior, CC5 fires rhythmically primarily to alternately shorten and relax the pedal arteries, perhaps to facilitate the large forward and backward movements of the buccal mass. During the appetitive phase of feeding, when the animal lifts its head from the substrate and extends its neck, the CC5 cells are inhibited, presumably, at least in part because of inhibitory inputs evoked by firing of C-PR.

In summary, the current findings support growing evidence for the existence of multifunctional neurons. For some behaviors or for certain aspects of behaviors, CC5 appears act either individually or as a functional pair and has relatively specific and dedicated functions. For other behaviors, CC5 appears to act more as an element of a distributed circuit and is neither necessary nor sufficient for any aspects of the behavior.

\section{REFERENCES}

Bablanian GM, Weiss KR, Kupfermann I (1987) Motor control of the appetitive phase of feeding behavior in Aplysia. Behav Neural Biol 48:394-407.

Barlow HB (1972) Single units and sensation: a neuron doctrine for perceptual psychology? Perception 1:371-394.

Bernheim SM, Mayeri E (1995) Complex behavior induced by egg-laying hormone in Aplysia. J Comp Physiol [A] 176:131-136.

Chiel HJ, Weiss KR, Kupfermann I (1986) An identified histaminergic neuron modulates feeding motor circuitry in Aplysia. J Neurosci 6:2427-2450.

Chiel HJ, Kupfermann I, Weiss KR (1988) An identified histaminergic neuron can modulate the outputs of buccal-cerebral interneurons in Aplysia via presynaptic inhibition. J Neurosci 8:49-63.

Churchland PS, Sejnowski TJ (1992) The computational brain. Cambridge, MA: MIT.

Cook DG, Carew TJ (1989) Operant conditioning of head-waving in Aplysia. I. Identified muscles involved in the operant response. J Neurosci 9:3097-3106.

Ferguson GP, Ter Maat A, Parsons DW, Pinsker HM (1989) Egg laying in Aplysia. I. Behavioral patterns and muscle activity of freely behaving animals after selectively elicited bag cell discharges. J Comp Physiol [A] 164:835-847.

Fetz EE (1992) Are movement parameters recognizably coded in the activity of single neurons? Behav Brain Sci 15:679-690.

Fredman SM, Jahan-Parwar B (1979) Intra- and interganglionic synaptic connections in the CNS of Aplysia. Brain Res Bull 4:393-406. 
Fredman SM, Jahan-Parwar B (1983) Command neurons for locomotion in Aplysia. J Neurophysiol 49:1092-1117.

Hening WA, Walters ET, Carew TJ, Kandel ER (1979) Motorneuronal control of locomotion in Aplysia. Brain Res 179:231-253.

Hooper SL, Moulins M (1989) Switching of a neuron from one network to another by sensory-induced changes in membrane properties. Science 244:1587-1589.

Jahan-Parwar B, Fredman SM (1979) Role of interganglionic synaptic connections in the control of pedal and parapodial movements in Aplysia. Brain Res Bull 4:407-420.

Koch UT, Koester J, Weiss KR (1984) Neuronal mediation of cardiovascular effects of food arousal in Aplysia. J Neurophysiol 51:126-135.

Kuenzi FM, Carew T (1991) Identification of neuronal pathways mediating phototactic modulation of head-waving in Aplysia californica. Behav Neural Biol 55:338-355.

Kuenzi FM, Carew TJ (1994a) Head waving in Aplysia californica. II. Functional anatomy and muscular activity during behaviour. J Exp Biol 195:53-74.

Kuenzi FM, Carew TJ (1994b) Head waving in Aplysia californica. I. Behavioural characterization of searching movements. J Exp Biol 195:35-51.

Kupfermann I (1970) Stimulation of egg laying by extracts of neuroendocrine cells (bag cells) of abdominal ganglion of Aplysia. J Neurophysiol 33:877-881.

Kupfermann I, Weiss KR (1978) The command neuron concept. Behav Brain Sci 1:3-39.

Kupfermann I, Weiss KR (1982) Activity of an identified serotonergic neuron in free moving Aplysia correlates with behavioral arousal. Brain Res 241:334-337.

Lockery SR, Kristan Jr WB (1990) Distributed processing of sensory information in the leech. II. Identification of interneurons contributing to the local bending reflex. J Neurosci 10:1816-1829.

McCaman RE, Weinreich D (1985) Histaminergic synaptic transmission the cerebral ganglion of Aplysia. J Neurophysiol 53:1016-1037.

Meyrand P, Simmers J, Moulins M (1994) Dynamic construction of a neural network from multiple pattern generators in the lobster stomatogastric nervous system. J Neurosci 14:630-644.

Morton DW, Chiel HJ (1994) Neural architectures for adaptive behavior. Trends Neurosci 17:413-420.

Nagahama T, Weiss KR, Kupfermann I (1993) Effects of cerebral neuron C-PR on body postural muscles associated with a food-induced arousal state in Aplysia. J Neurophysiol 70:1231-1243.

Ono JK, McCaman RE (1980) Identification of additional histaminergic neurons in Aplysia: improvement of single cell isolation techniques for in tandem physiological and chemical studies. Neuroscience 5:835-840.
Rosen SC, Weiss KR, Goldstein RS, Kupfermann I (1989) The role of a modulatory neuron in feeding and satiation in Aplysia: effects of lesioning of the serotonergic metacerebral cells. J Neurosci 9:1562-1578.

Rosen SC, Teyke T, Miller MW, Weiss KR, Kupfermann I (1991) Identification and characterization of cerebral-to-buccal interneurons implicated in the control of motor programs associated with feeding in Aplysia. J Neurosci 11:3630-3655.

Segal MM, Koester J (1982) Convergent cholinergic neurons produce similar postsynaptic actions in Aplysia: implications for neural organization. J Neurophysiol 47:742-759.

Skelton ME, Koester J (1992) The morphology, innervation and neural control of the anterior arterial system of Aplysia californica. J Comp Physiol [A] 171:141-155.

Susswein AJ, Gev S, Achituv Y, Markovich S (1984) Behavioral patterns of Aplysia fasciata along the Mediterranean coast of Israel. Behav Neural Biol 41:7-22.

Teyke T, Weiss KR, Kupfermann I (1990a) Appetitive feeding behavior of Aplysia: behavioral and neural analysis of directed head turning. J Neurosci 10:3922-3934.

Teyke T, Weiss KR, Kupfermann I (1990b) An identified neuron (C-PR) evokes neuronal responses reflecting food arousal in Aplysia. Science 247:85-87.

Teyke T, Weiss KR, Kupfermann I (1991) Activity of identified cerebral neuron correlates with food-induced arousal in Aplysia. Neurosci Lett 133:307-310.

Weiss KR, Kupfermann I (1976) Homology of the giant serotonergic neurons (metacerebral cells) in Aplysia and pulmonate molluscs. Brain Res 117:33-49.

Weiss KR, Cohen JL, Kupfermann I (1978) Modulatory control of buccal musculature by a serotonergic neuron (metacerebral cell) in Aplysia. J Neurophysiol 41:181-203.

Weiss KR, Shapiro E, Kupfermann I (1986) Modulatory synaptic actions of an identified histaminergic neuron on the serotonergic metacerebral cell of Aplysia. J Neurosci 6:2393-2402.

Wu J-Y, Cohen LB, Falk CX (1994) Neuronal activity during different behaviors in Aplysia: a distributed organization? Science 263:820-823.

Xin Y, Kupfermann I (1995) Neuronal pathways mediating head turning behavior in Aplysia. Neurosci Lett 186:197-199.

Xin Y, Weiss KR, Kupfermann I (1996) A pair of identified interneurons in Aplysia that are involved in multiple behaviors are necessary and sufficient for the arterial shortening component of a local withdrawal reflex. J Neurosci 16:4518-4528. 